\title{
Un exemple de difficultés de la protection du littoral contre l'érosion marine: le cas de la côte d'Anglet (Pyrénées Atlantiques)
}

\author{
Protection of the Anglet coast against marine erosion
}

\author{
$M^{\text {me }}$ Solange Manoujian \\ Ingénieur
}

\author{
Claude Migniot \\ Ingénieur en Chef
}

Département Sédimentologie du Laboratoire Central d'Hydraulique de France

Le littoral du Pays Basque, situé au fond du Golfe de Gascogne, occupe une place privilégiée pour la puissance de ses houles puisque c'est sur cette partie de nos côtes françaises que l'on rencontre les vagues les plus fortes et, de ce fait, les pouvoirs de destruction les plus importants.

Associée à un déficit sédimentaire depuis plusieurs décennies, cette force des houles a entrainé une dégradation progressive des fonds marins et un recul spectaculaire de la ligne de côte que l'on retrouve tout le long du littoral depuis l'embouchure de la Gironde jusqu'à la frontière d'Espagne. Sept cents à huit cents mètres de recul de la laisse de basse-mer à Cap Breton entre 1881 et 1922, un à trois mètres par an sur la côte des Landes, là 2,5 mètres par an à Saint Jean de Luz entre 1864 et 1877, 20 mètres de recul des falaises de Biarritz entre 1938 et 1969 soit $0,60 \mathrm{~m}$ par an, 3 à 4 mètres d'approfondissement des fonds de $-7 \grave{a}-3 \mathrm{~m}$ entre 1826 et 1953 devant Chiberta et des reculs de plus de 10 métres par an à Seignosse et en certains points de la côte d'Anglet, sont des chiffres qui montrent toute l'importance des érosions depuis plus d'un siècle.

Phénomènes naturels inéluctables exacerbés par les interventions humaines multiples allant depuis les emprunts de sable jusqu'aux ouvrages d'amélioration des plages ou des accès aux ports de Bayonne et de Cap Breton, un fait reste certain c'est que, quelles que soient les causes, la côte est l'objet d'une érosion générale que l'on retrouve au Sud de l'embouchure de l'Adour dans la zone du district de Bayonne - Anglet - Biarritz.

Devant la gravité du problème qui se pose le long de la côte d'Anglet et de la côte des Basque des études ont été entreprises au Laboratoire Central d'Hydraulique de France (L.C.H.F.) à la demande des collectivités locales, de la Direction Départementale de l'Equipement des Pyrénées Atlantiques (D.D.E.) et de la Mission Intermi- nistérielle d'Aménagement de la côte d'Aquitaine (M.I.A.C.A.) pour :

- préciser les causes exactes des phénomènes de dégradation,

- prévoir ce qui se passerait si on laissait la nature libre de ses actions,

- rechercher des solutions permettant de réduire l'importance des érosions et dans toute la mesure du possible redonner à toute cette région son potentiel balnéaire et touristique.

En étudiant très attentivement l'ensemble des documents antérieurs, en réalisant des mesures hydrographiques, océanographiques et sédimentologiques, en utilisant les techniques modernes des traceurs fluorescents et radioactifs, il a été possible de bien cerner la réalité et de quantifier les phénomènes naturels.

En procédant à des études sur les modèles réduits de la côte d'Anglet et de la côte des Basques on a pu examiner l'efficacité de différentes solutions de protection, éliminer celles qui n'étaient pas applicables à la force des houles du Golfe de Gascogne, retenir celles qui pouvaient apporter une amélioration sensible sans entrainer des dépenses hors de proportion avec le but poursuivi.

Les phénomènes naturels devant le littoral d'Anglet

\section{Houles - marées et sédiments}

En abordant ce problème il faut insister sur l'importance des houles de cette partie du littoral français et sur leur puissance dévastatrice. Pendant 50 jours par an l'amplitude maximale $\mathrm{H} \mathrm{1/10}$ dépasse $3,75 \mathrm{~m}$, pendant 10 jours par an $5,50 \mathrm{~m}$, pendant 2 jours par an $7 \mathrm{~m}$ et 

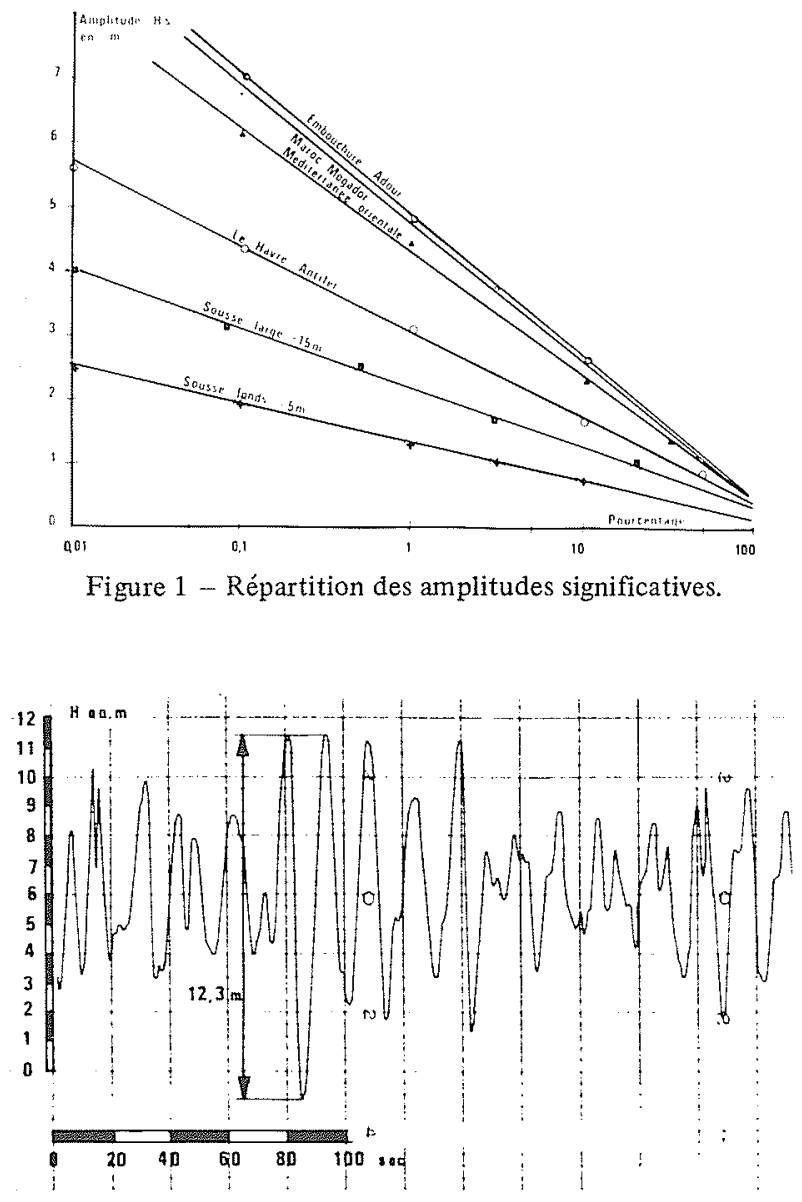

Figure 2 - Enregistrement au large du phare de Biarritz..

un jour tous les 10 ans elle sera supérieure à $10 \mathrm{~m}$. La tempête de Décembre 1976 a donné des amplitudes de $12,30 \mathrm{~m}$ et de 16 secondes de période qui montrent bien l'importance des facteurs océanographiques (Figures 1 et 2).

A ces vagues de grande puissance on devra adapter des ouvrages de protection appropriés qui, non seulement, atténuent les amplitudes sur le littoral mais résistent sans dommage appréciable aux tempêtes répétées de l'hiver ; le déplacement des blocs de 50 tonnes de la digue de Socoa et certaines dégradations survenues aux blocs de 40 tonnes de la digue de l'Adour sont les meilleurs exemples incitant à la prudence dans les travaux à la mer.

Le marnage appréciable, pouvant atteindre une amplitude de $4.85 \mathrm{~m}$ par marée de vives eaux exceptionnelles, sera le deuxième paramètre dont il faudra tenir compte pour la défense du littoral, ce qui conduira à des ouvrages relativement hauts pour ne pas être immergés par les eaux et pour pouvoir être réalisables par les Entreprises.

Si les matériaux des plages sont constitués de deux stocks sédimentaires comportant des éléments grossiers (madrague) mélangés à des sables plus fins, les sédiments des petits fonds sont des éléments très fins de 0,15 à $0,20 \mathrm{~mm}$ de diamètre. Ce troisième paramètre ne facilite pas la stabilité des plages et entraine des déplacements importants que l'on a estimés par kilomètre de plage à près de $500000 \mathrm{~m}^{3}$ par an perpendiculairement à la côte, avec des mouvements alternatifs de départ vers le large et de retour vers la côte, ainsi que des déplacements parallèlement au littoral, soit vers le Nord, soit vers le Sud, dont le volume total atteint près de $900000 \mathrm{~m}^{3}$ par an avec un déficit annuel important.

\section{L'érosion du littoral d'Anglet}

La côte d'Anglet n'échappe pas à l'érosion générale du littoral et l'on peut même avancer que, par suite de sa position géographique, la régression de la côte prend un caractère d'autant plus alarmant que cette zone est indispensable au développement touristique et balnéaire du Pays Basque et plus particulièrement du district Bayonne - Anglet - Biarritz.

Comme pour l'ensemble du littoral du Golfe de Gascogne on constate tout d'abord, et ceci sans aucune équivoque, que le littoral d'Anglet était en voie d'érosion dans toute la première moitié du siècle, c'est-à-dire avant que la digue Nord de protection de l'embouchure n'ait èté réalisée.

Entre 1826 et 1953 les sondages du Service Hydrographique de la Marine accusaient déjà des érosions pouvant atteindre 3 à $4 \mathrm{~m}$ dans les petits fonds de $-7 \grave{a}-3 \mathrm{~m}$; on remarquait également que sous l'action des courants de l'Adour canalisés par le "convergent" et des dragages, une partie des matériaux était entrainée vers le large par des fonds de $15 \mathrm{~m}$ et plus où ils ne pouvaient être repris que très partiellement par les houles. On peut évaluer à $200000 \mathrm{~m}^{3}$ par an les volumes des matériaux érodés devant le côte d'Anglet à cette époque, ce volume ne prenant pas en compte la totalité du littoral d'Anglet.

Au cours de cette même période on notait un recul de la laisse de pleine mer de $50 \mathrm{~m}$ entre 1898 et 1959 à $400 \mathrm{~m}$ au Sud de la jetée Sud. Entre 1952 et 1963, c'està-dire au cours de la décennie qui a précédé la construction de la digue Nord, le trait de côte a reculé de 3 à $4 \mathrm{~m}$ par an en moyenne sur une longueur de plus de $2 \mathrm{~km}$ au Sud de l'embouchure ; au-delà l'examen des délimitations du domaine maritime au 15 décembre 1931 et au 15 mars 1954 conduirait à une érosion de 50 à $75 \mathrm{~m}$, c'est-à-dire de $3 \mathrm{~m}$ par an.

Ces résultats sont confirmés par les photographies aériennes effectuées par l'Institut Géographique National en 1938,1954 et 1962.

Entre 1953 et 1973 la comparaison des sondages montre que le littoral au Nord de l'embouchure ne s'est modifié que faiblement ; d'une façon très générale, sur une distance de $2500 \mathrm{~m}$ l'estran s'est érodé de $700000 \mathrm{~m}^{3}$ environ et les petits fonds marins sédimentés de $350000 \mathrm{~m}^{3}$, le bilan était donc négatif. Audelà des fonds de $-10 \mathrm{~m}$ les mouvements sédimentaires sont négligeables.

$\mathrm{Au}$ Sud de l'embouchure les mouvements sont beaucoup plus importants et s'étendent sur toute une zone de $800 \mathrm{~m}$ à $1000 \mathrm{~m}$ de largeur où l'on observe des érosions pouvant atteindre 4 à 5 millions de $\mathrm{m}^{3}$ soit près de $500000 \mathrm{~m}^{3}$ par an. Au large on retrouve une importante zone de sédimentation dépassant $10 \mathrm{~m}$ d'épaisseur provenant du dépôt des produits de dragages effectués dans le chenal d'accès. 


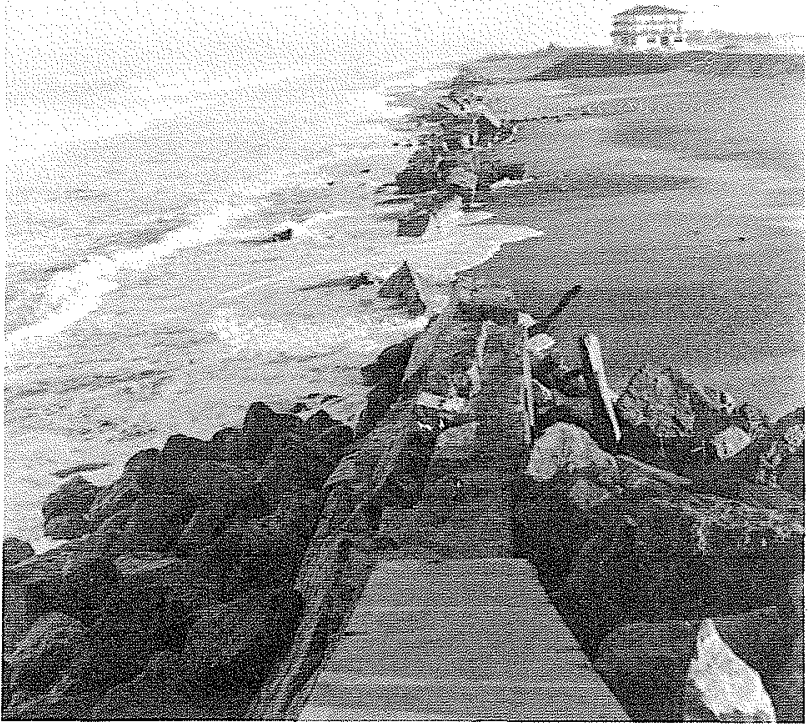

Figure 3 - Erosion de la côte au Nord des Sables d'Or.

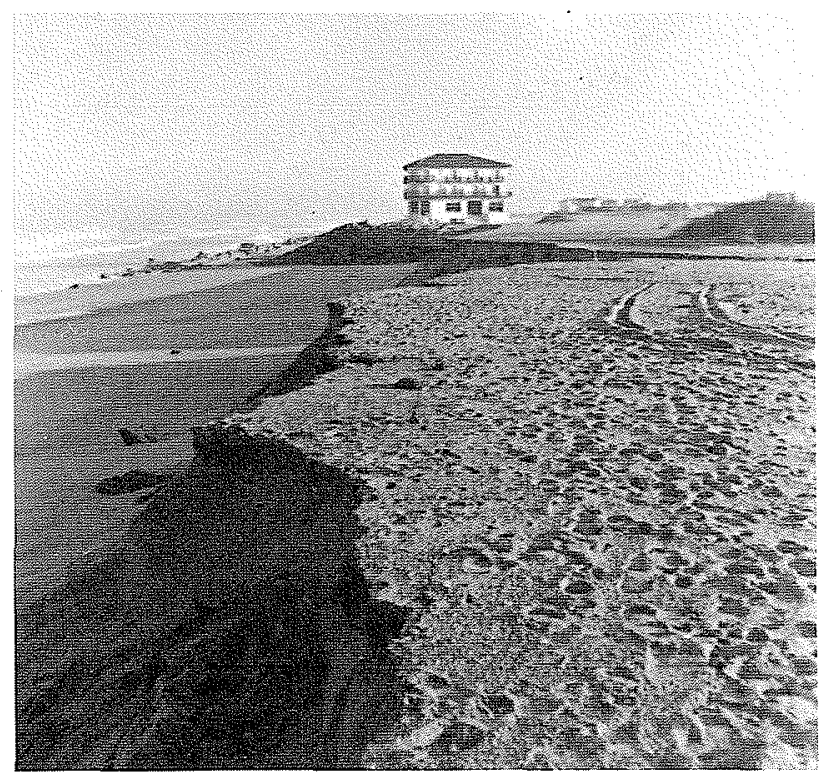

Figure 4 - Destruction du littoral aux abords de Marinella.

La comparaison des profils d'estran levés presque chaque année montre une avancée du littoral immédiatement au Sud de la petite jetée Sud, c'est-à-dire dans la zone protégée par la digue Nord. Plus au Sud la côte aurait reculé de $40 \mathrm{~m}$ en moyenne entre 1963 et 1973 , soit $4 \mathrm{~m}$ par an, le maximum de régression se situant en 1973 à $2300 \mathrm{~m}$, avec 4,70 m par an, les érosions gagnant au cours des temps de plus en plus vers le Sud.

Les petits fonds marins peuvent s'éroder de 3 à $4 \mathrm{~m}$ dans certains secteurs entre les fonds de 0 et $-7 \mathrm{~m}$ et restent à peu près stables -à l'exclusion des zones de dépôts - au-delà de $-10 \mathrm{~m}$. En général on remarque que les fonds sont littéralement "tronqués" par les érosions à une cote voisine de -4 à $-6 \mathrm{~m}$.
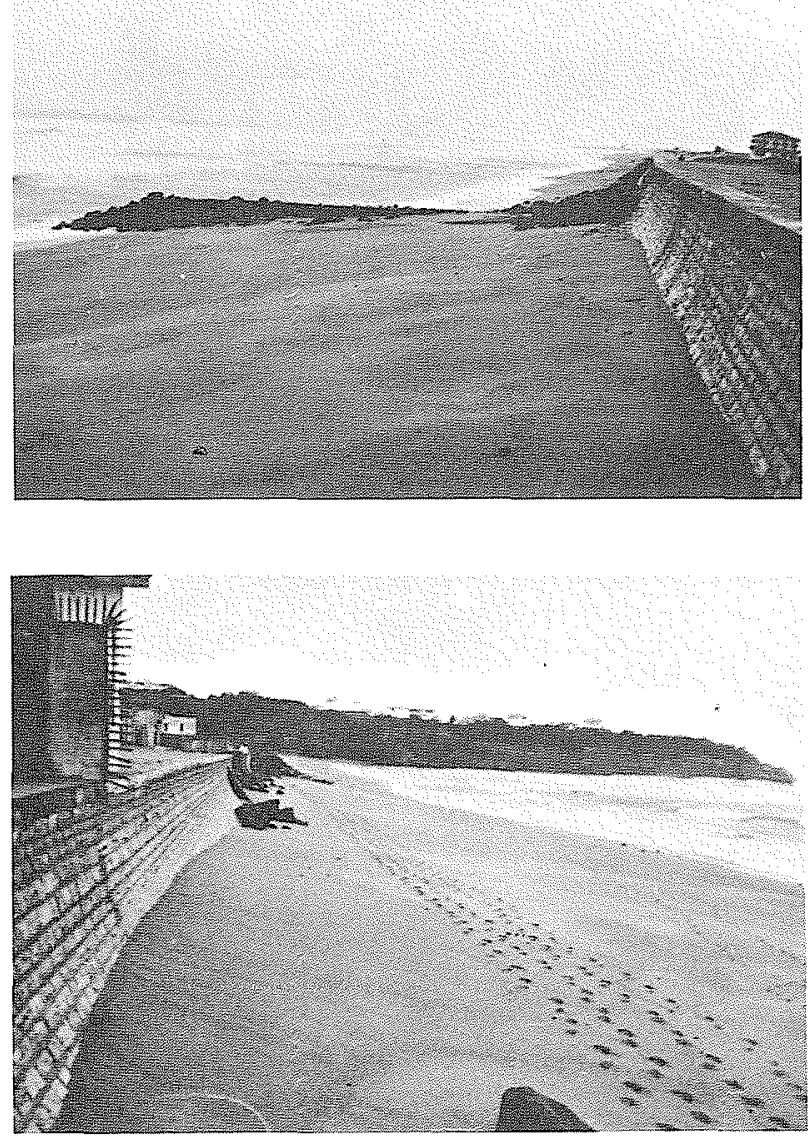

Figures 5, 6 - Etat des plages devant les Sables d'Or avant 1974.

En 1973 et 1974 des dégâts spectaculaires apparaissaient dans toute la partie urbanisée située au Sud de la côte d'Anglet. Le mur de protection de l'ancienne route littorale continuait à se dégrader (Figure 3) et 1'hôtel Marinella était menacé (Figure 4). Devant les Sables d'Or la plage qui existait encore quelques mois plus tôt (Figures 5 et 6) disparaissait entièrement, en Décembre 1974, entrainant le déchaussement des ouvrages du front de mer et de ses palplanches (Figures 7 et 8 ) ; l'abaissement du niveau de la plage atteignait près de 4 m (Figure 9) vers la même époque, en Février 1975, l'érosion gagnait l'extrémité Sud située devant le Village de Vacances détruisant les parkings (Figure 10), et menaçant le grand immeuble du V.V.F. (Figure 11).

A cette époque la perte annuelle en sédiments était estimée entre 100 et $150 \mathrm{~m}^{3}$ par mètre linéaire de plage, le recul du trait de côte pouvant atteindre en certains points 10 à 15 mètres par an conduisant à brève échéance à la destruction totale de cette partie de notre littoral y compris toute la zone urbanisée.

\section{La protection du littoral d'Anglet - Chiberta entre l'embouchure de l'Adour et le Cap St Martin}

Devant l'ampleur des phénomènes d'érosion qui menaçait le littoral d'Anglet, des études ont été effec- 

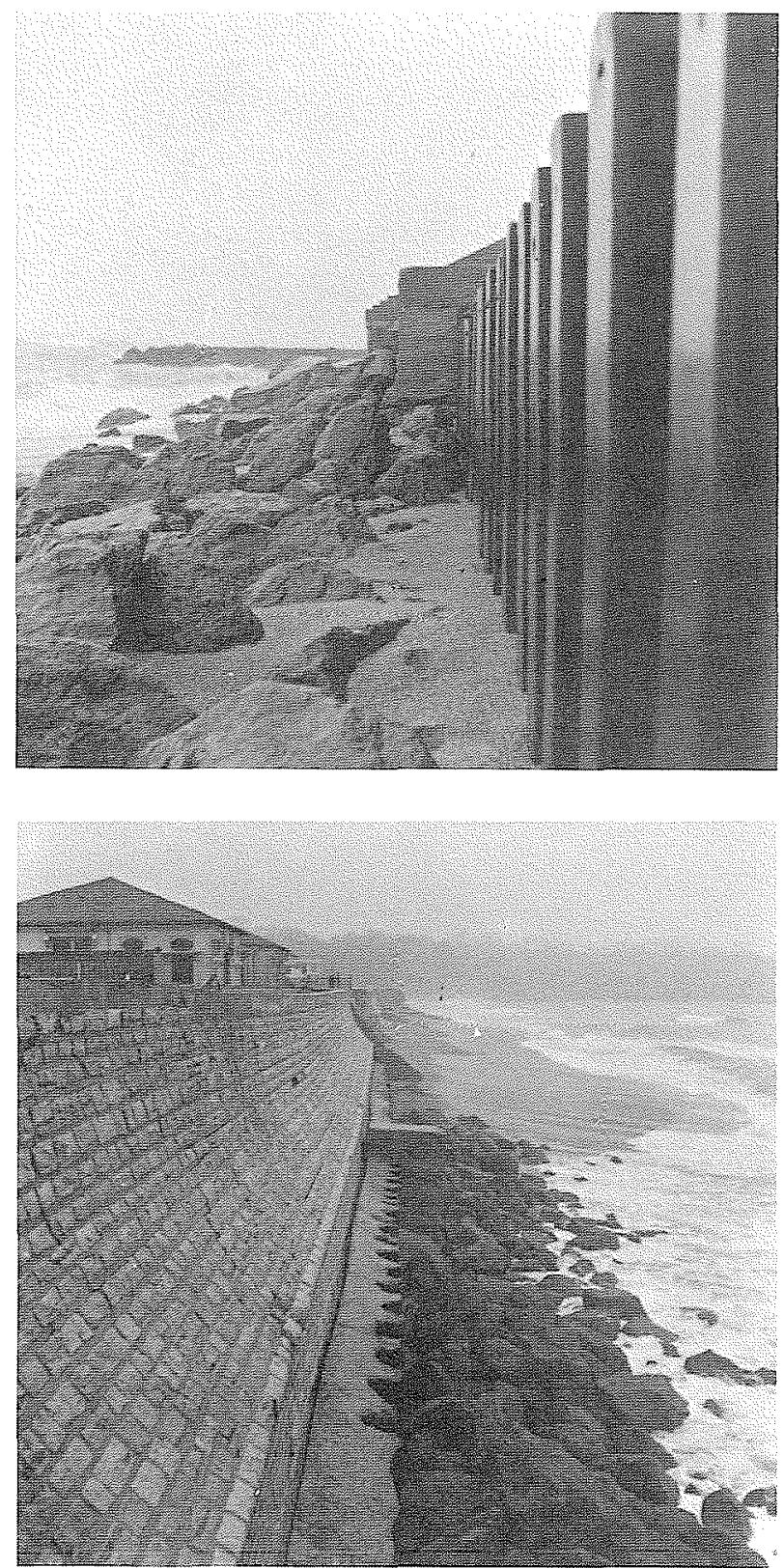

Figures 7,8 - Dégradation devant les Sables d'Or le 2 décembre 1974.

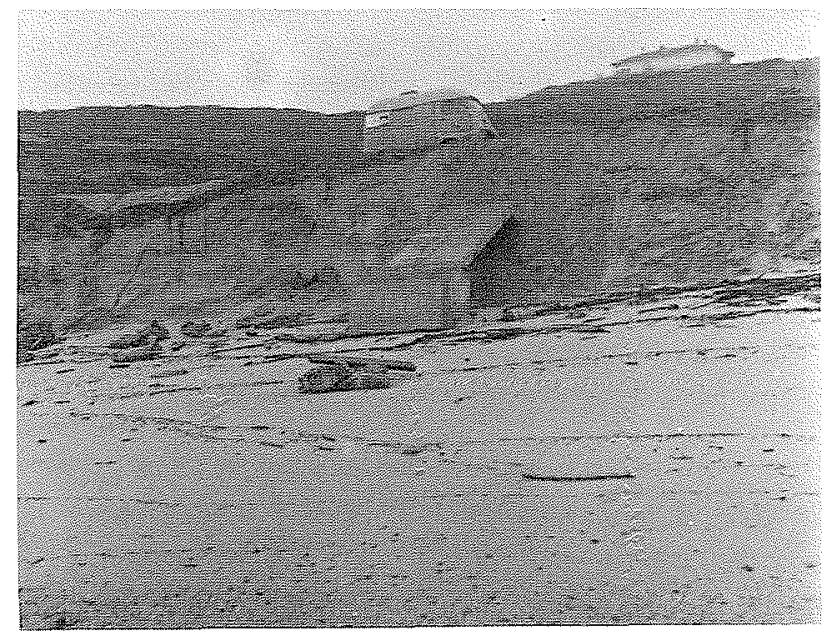

Figure 9 - Erosion du littoral au Nord immédiat du VVF.

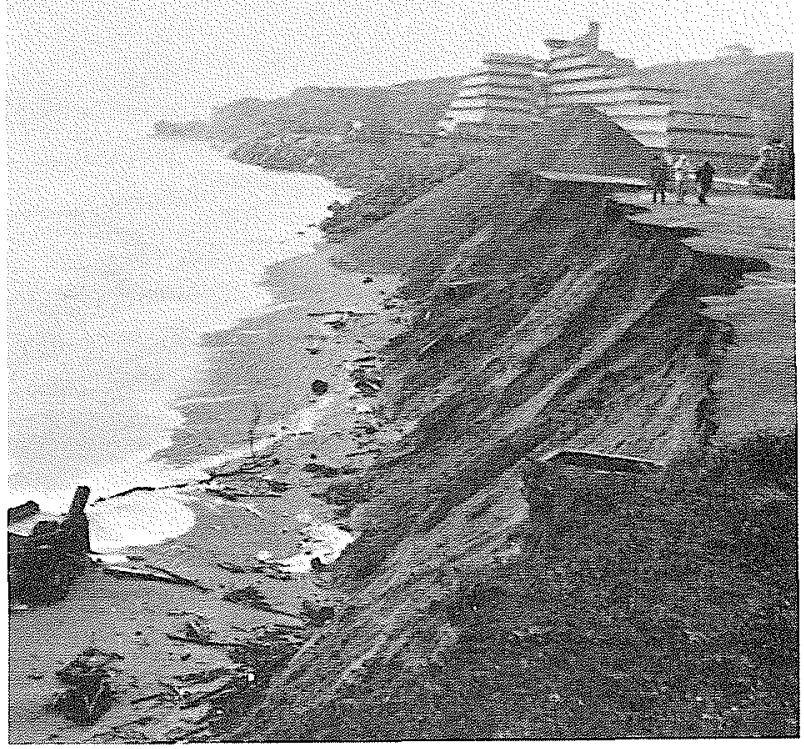

Figure 10 - Destruction du parking devant le VVF.

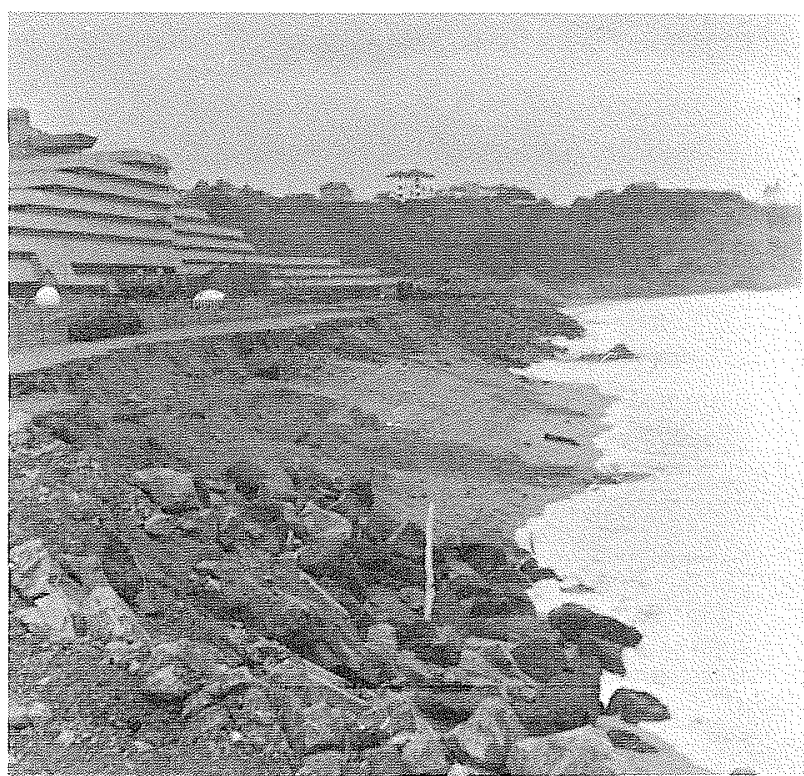

Figure 11 - Erosion devant le VVF Mars 1975.

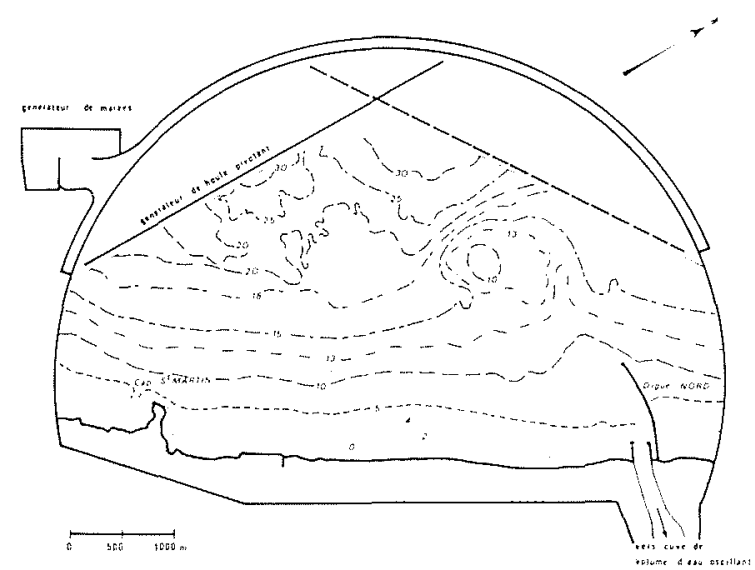

Figure 12 - Plan du modèle. 


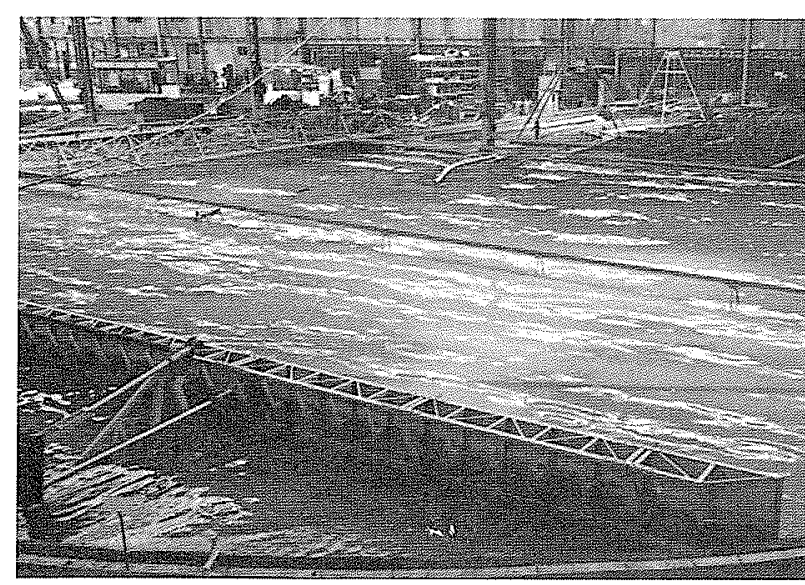

Figure 13 - Vue générale du modèle et son générateur de houle.

tuées en 1974-1975 sur un modèle réduit à l'échelle du $1 / 200$ e en plan et $1 / 75$ en hauteur (Figures 12 et 13$)$.

$\mathrm{Ce}$ modèle représentait très fidèlement le littoral et les fonds depuis une zone située à 500 mètres au Nord de l'embouchure de l'Adour jusqu'au-delà du Cap St Martin. Il s'étendait vers le large jusqu'aux profondeurs de $25 \mathrm{~m}$ et représentait très exactement les différents sédiments naturels (sables, madrague...) à l'aide de matériaux artificiels convenablement broyés et répartis sur le modèle sur une épaisseur suffisante pour représenter correctement les possibilités d'érosion.

Un générateur de houles pivotant permettait de réaliser, en continu, toutes les fluctuations d'amplitudes, de périodes et de directions qui avaient été relevées en nature ainsi que les houles en "trains d'ondes" que l'on constate dans ce secteur. La durée d'action de chaque houle était reproduite à une échelle des temps "sédimentologiques" du $1 / 500 \mathrm{e}$, une année naturelle étant ainsi représentée en 6 heures environ sur le modèle et une tempête de 3 à 4 jours en moins de 4 minutes.

Un générateur de marée donnait les fluctuations du niveau de l'eau sous l'effet des marées et entrainait en plus le remplissage et le vidage de l'Adour ainsi que la formation des courants de flot et de jusant liés à ces phénomènes.

De même les crues de l'Adour qui peuvent dépasser $2000 \mathrm{~m}^{3} / \mathrm{s}$ étaient réalisées avec toute la précision désirable.

Sur cette vaste machine analogique il était possible de représenter le passé, de définir les causes des érosions, de prévoir l'avenir et de rechercher les ouvrages à construire pour sauvegarder ce patrimoine touristique que représente la côte d'Anglet.

Après avoir constaté que le modèle réduit respectait très exactement les évolutions des fonds connues au cours des dernières années et en particulier les fluctuations des profils des plages, l'érosion générale des petits fonds, le recul du trait de côte (Figure 14), la destruction du mur de soutènement de la route littorale qui passait devant l'Hòtel Marinella (Figure 15), les apports dans l'embouchure de l'Adour, et d'une façon générale le déficit littoral que l'on a observé en nature,

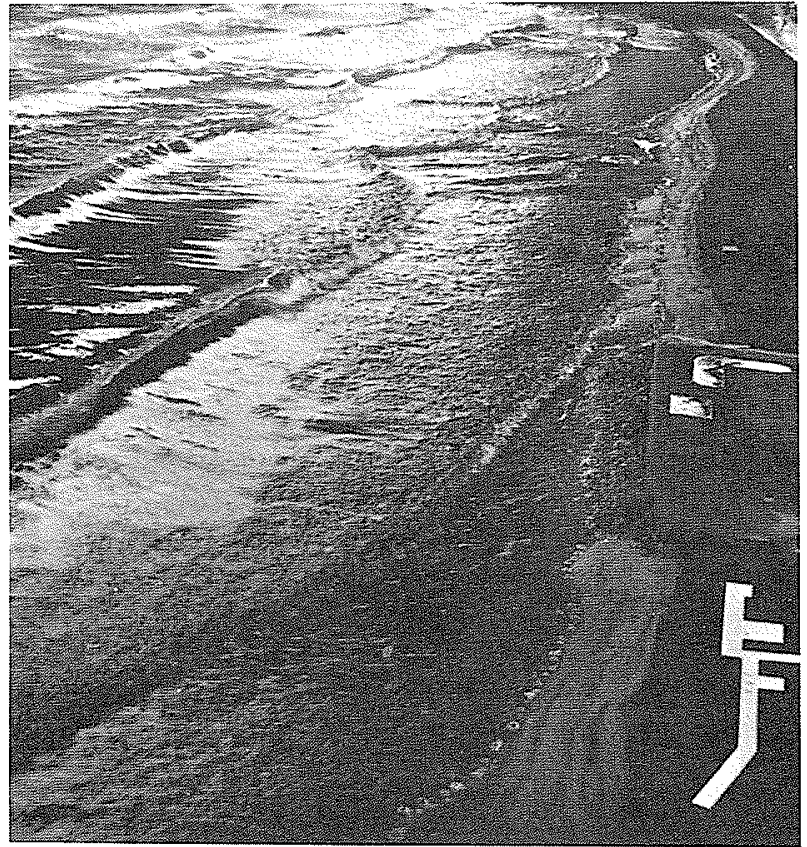

Figure 14 -- Erosion générale de la côte sur le modèle.

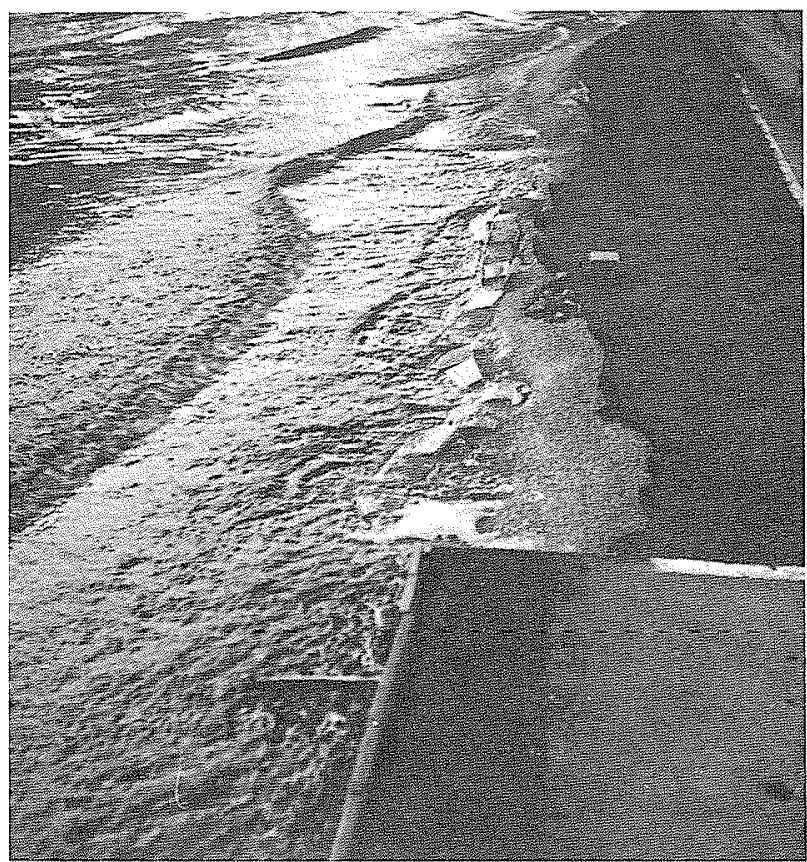

Figure 15 - Destruction du mur de soutènement et érosion devant Marinella sur le modèle.

il a été possible d'examiner quelles seraient les évolutions des fonds au cours des prochaines années.

Sans ouvrage de défense on constate tout d'abord que l'érosion croît pratiquement linéairement lorsque l'on s'éloigne vers le Sud où elle atteint son paroxysme. Les fonds marins sont, sur le modele, véritablement "rabotés" par les houles à des cotes voisines de -4 à $-6 \mathrm{~m}$ où un plateau de $300 \mathrm{~m}$ de largeur apparait. Les vagues n'étant plus freinées par les petits fonds viennent attaquer directement l'estran qui se dégrade avec une grande rapidité. 


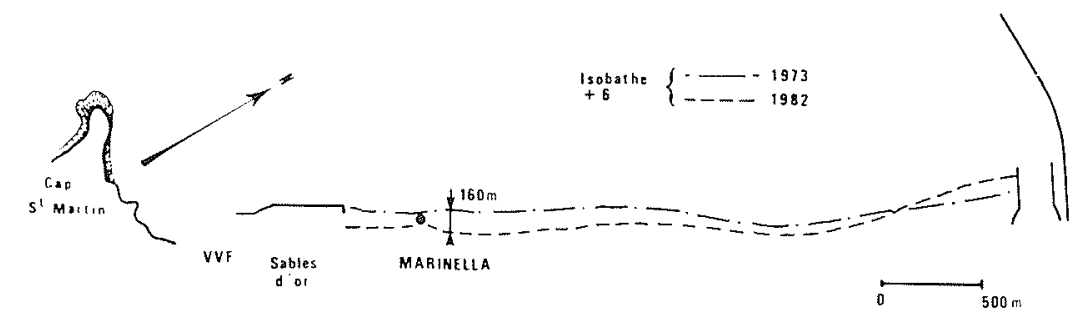

Figure 16 - L'érosion du littoral en 1982 si aucun ouvrage n'avait été réalisé (modèle).

Malgré des déversements de produits de dragages de $160000 \mathrm{~m}^{3}$ par an entre les fonds de $-8 \mathrm{a}-10 \mathrm{~m}$ le trait de côte aurait reculé de $7 \mathrm{~m}$ par an à $2000 \mathrm{~m} \mathrm{au}$ Sud de la petite jetée Sud, $10 \mathrm{~m}$ à $2400 \mathrm{~m}, 11 \mathrm{~m}$ à $2800 \mathrm{~m}$ et $14 \mathrm{~m}$ par an entre 3200 et $3600 \mathrm{~m}$, c'est-àdire dans toute la zone des Sables d'Or et de Marinella.

En 1980-1982 si aucun ouvrage n'avait été réalisé la laisse de pleine-mer, qui est caractérisée en nature par la limite atteinte en pleine-mer de vives-eaux, se situerait à 120 mètres environ en arrière de l'Hôtel Marinella qui deviendrait un îlot isolé de la terre. Le mur de protection des Sables d'Or serait contourné et le Golf très endommagé. Par contre aux abords mêmes de la petite jetée Sud de l'embouchure, les fonds resteraient stables, les sables et la madrague de la côte d'Anglet continuant à s'ébouler dans le chenal de navigation à chaque tempête et à l'obstruer (Figure 16).

En extrapolant les résultats des essais à l'état présumé de 1990 on constate que l'ensemble du littoral de Chiberta - Anglet aurait reculé de $150 \mathrm{~m}$ en moyenne sur toute la zone qui s'étend depuis le village de vacances (V.V.F.) jusqu'à plus de $1500 \mathrm{~m}$ au Nord des Sables d'Or. Au-delà les érosions diminueraient progressivement pour s'annuler aux abords de l'Adour.

Pour préciser encore l'ampleur de l'érosion signalons que le modèle indique, malgré les dépôts de dragages, une déperdition devant la côte d'Anglet de $370000 \mathrm{~m}^{3}$ par an, ce qui représente plus de 3 millions de $\mathrm{m}^{3}$ de sédiments qui auraient disparu des petits fonds et de la plage entre 1973 et 1982 .

Cet accroissement de l'érosion au Sud du littoral s'est trouvé malheureusement confirmé en nature, nécessitant des interventions immédiates de l'Administration pour protéger les installations existantes du Village de Vacances, des Sables d'Or et de Marinella. Les photographies prises devant les Sables d'Or, le Village de Vacances et l'ensemble de la côte montrent ce qu'était le littoral à cette époque et laissent présumer ce qu'il aurait été si des travaux de défense n'avaient été entrepris.

Pour évaluer l'influence des ouvrages de protection mis en œuvre il faudra toujours se rappeler cet "état de référence" du littoral non aménagé et l'état catastrophique du Village de Vacances et des Sables d'Or dès 1974.

La protection du littoral d'Anglet - Chiberta a porté sur différentes solutions comportant soit des épis droits plus ou moins espacés, soit des épis en $T$ formant briselames, soit une combinaison entre ces différents types d'ouvrages.
Dans tous les cas, quelle que soit la solution adoptée pour les ouvrages, les essais effectués en modèle réduit ont montré qu'il fallait les associer à des rechargements de plages avec des matériaux appropriés comportant un mélange de sables grossiers et de madrague (30\% de graviers) et qu'il était nécessaire de continuer à déverser des produits de dragages dans la zone des petits fonds pour compenser les déficits sédimentaires accumulés au cours des temps.

Avec de telles solutions il était possible de freiner l'érosion du littoral et de tendre vers un certain état d'équilibre tout au moins dans la zone dite "urbanisée" qui s'étend dans la partie Sud de la côte, la partie centrale devant rester, pour des raisons économiques, à l'état de "plage sauvage" soumise à des érosions appréciables.

Compte tenu des impératifs financiers la solution finalement retenue pour protéger le secteur Sud de la plage d'Anglet, les abords de l'hippodrome et du chenal d'accès au Port de Bayonne, comporte :

- 4 épis droits atteignant les fonds de 0 à $-1 \mathrm{~m}$ et réalisés respectivement au centre des Sables d'Or, entre les Sables d'Or et Marinella, à $175 \mathrm{~m}$ au Nord de Marinella, enfin au Sud des Sables d'Or vers le V.V.F. Ce dernier épi n'avait que $100 \mathrm{~m}$ de longueur alors que les trois autres devaient dépasser l'alignement du mur des Sables d'Or de $135 \mathrm{~m}$ vers le large ;

- une digue de $400 \mathrm{~m}$ de longueur située au Sud immédiat de l'embouchure de l'Adour et destinée à protéger l'embouchure de l'Adour ; cette digue devait atteindre les fonds de $-6 \mathrm{~m}$ pour etre efficace ;

- des rechargements en madrague entre les 4 épis et portant sur un déversement total de $210000 \mathrm{~m}^{3}$ de matériau (Figure 17) ;

- enfin des déversements de produits de dragages, provenant du Port de Bayonne, et répartis à raison de $200000 \mathrm{~m}^{3}$ par an dans la zone des petits fonds situés devant les Sables d'Or et Marinella.

Avec les aménagements retenus pour tenir compte des impératifs économiques de défense du littoral et par comparaison avec les évolutions des fonds que l'on aurait constatées sans aménagements les résultats du modèle permettaient de donner les conclusions suivantes :

- la digue Sud de l'Adour atteignant les fonds de $-6 \mathrm{~m}$ (fonds 1971) doit permettre de réduire progressivement les apports dans le chenal d'accès au Port de Bayonne au tiers de ce qu'ils étaient au cours des dernières années. L'efficacité de la digue se maintient pendant plus de dix ans, elle améliore également 

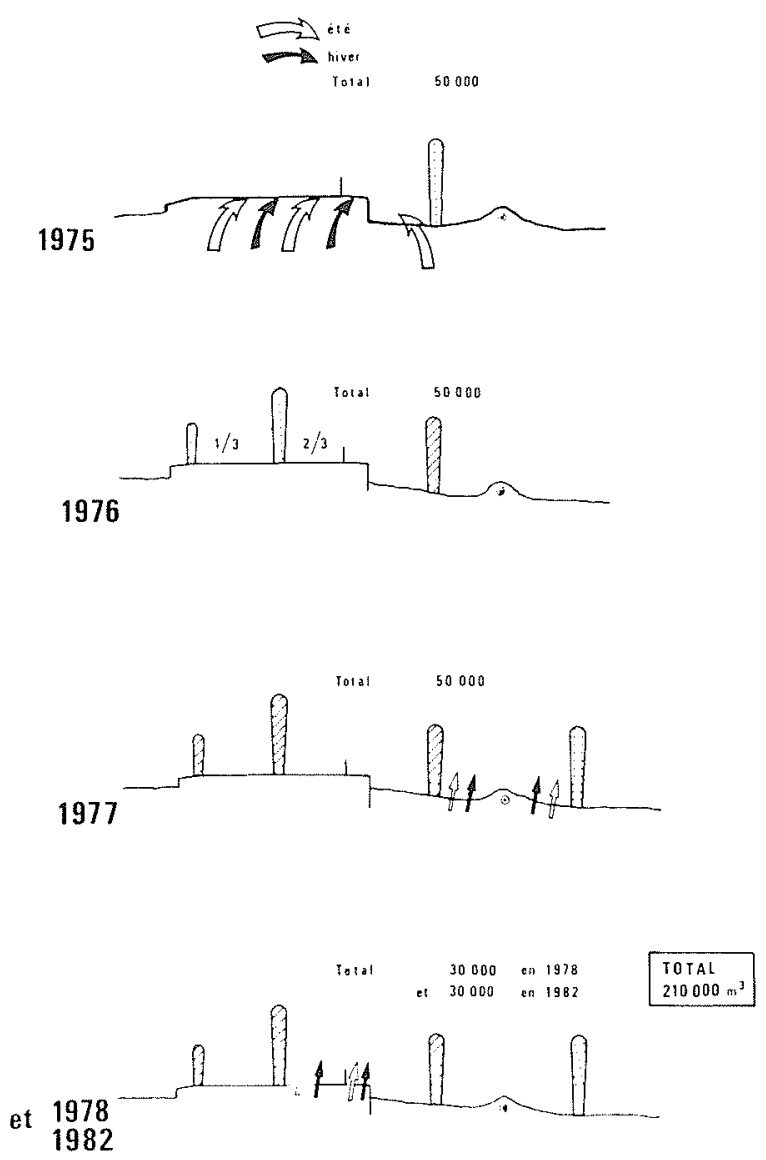

Figure 17 - Protection du littoral d'Anglet sur le modèle jusqu'en 1982 .

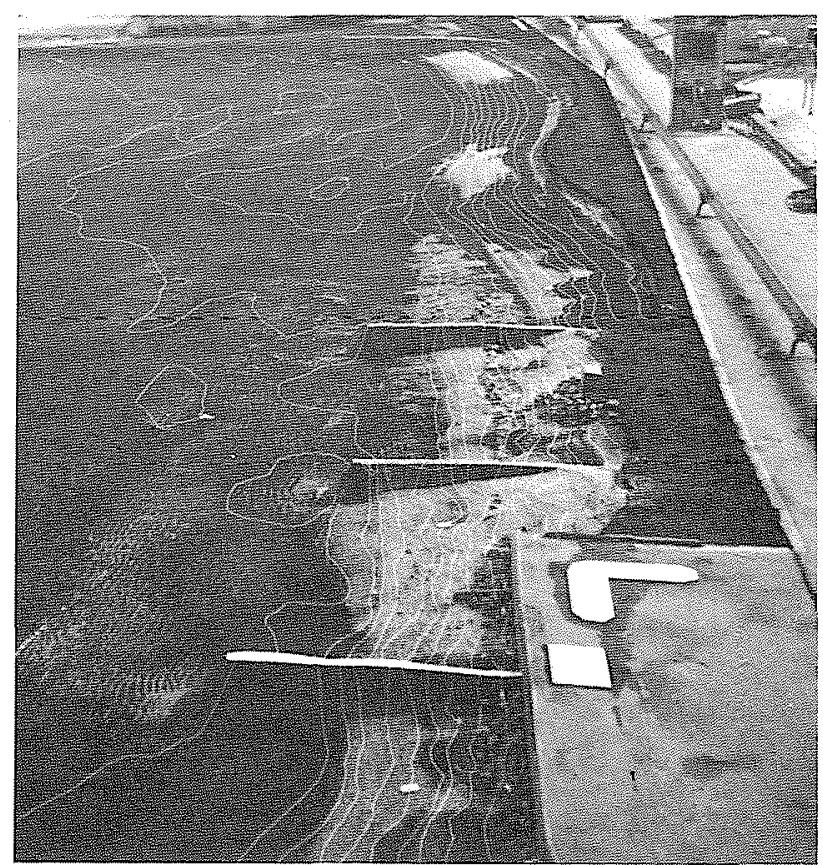

Figure 18 - Côte protégée dans la zone urbanisée des Sables d'Or (modèle).

la navigation par suppression du courant transversal qui avait tendance à "couper" le chenal au cours des tempêtes ;

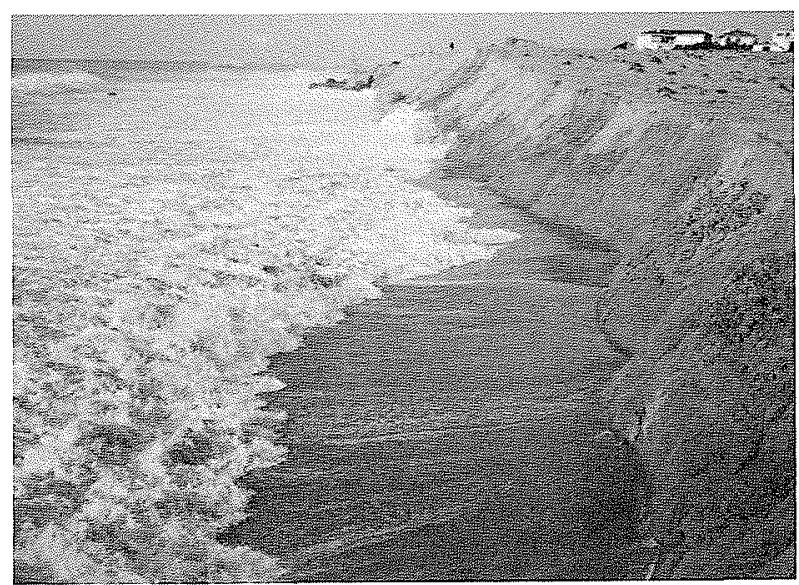

Figure 19 - Au Nord de Marinella dans la zone dite "plage sauvage" la côte continue de s'éroder en nature (18 mars 1976).

- au Sud immédiat de la digue de $400 \mathrm{~m}$ la côte sera stabilisée ou en légère sédimentation sur une distance de $1 \mathrm{~km}$ environ permettant de supprimer l'importante érosion actuellement observée ; par contre, immédiatement au Nord on devrait observer un léger recul du littoral ;

-- dans la zone dite "plage sauvage" située entre l'épi Nord de Marinella et la limite précédente, c'està-dire sur $1500 \mathrm{~m}$ environ, la côte continuera à s'éroder, le recul étant d'autant plus important que l'on se rapproche de Marinella. Ce recul du littoral restera toutefois moins fort que ce qu'il aurait été dans l'état actuel et diminuera au cours du temps; il peut cependant atteindre 10 à $12 \mathrm{~m}$ par an devant le Golf (Figures 18-19);

- dans la zone urbanisée située au Sud de Marinella, et englobant les Sables d'Or et le dispositif de protection par des épis avec rechargement en madrague et produits de dragage, l'érosion de l'estran est prati-

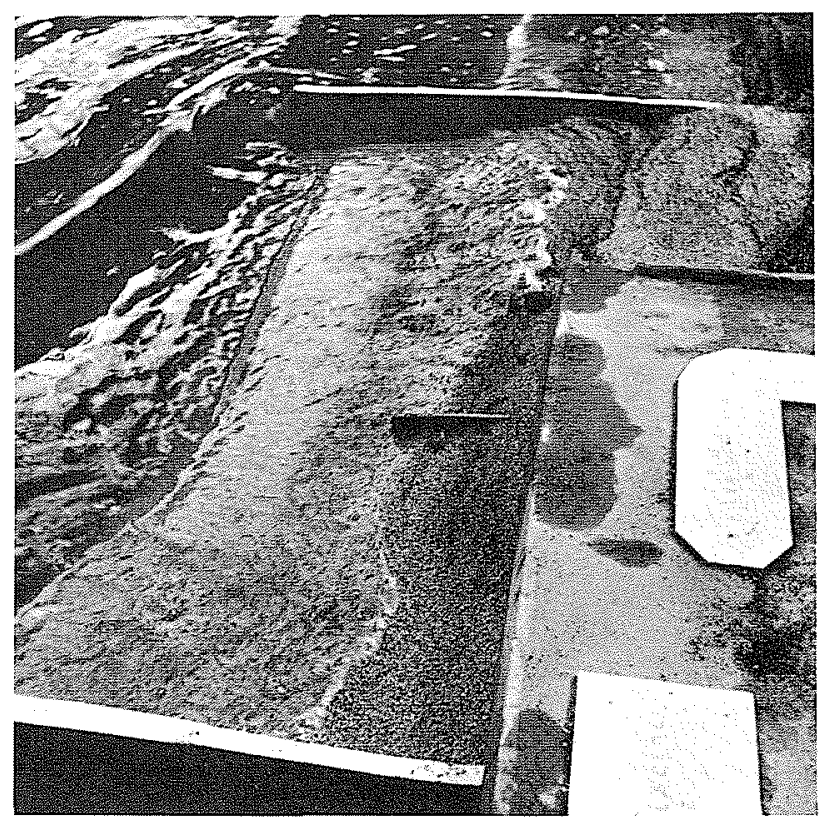

Figure 20 - Reformation d'une plage devant et au Nord des Sables d'Or (modèle). 


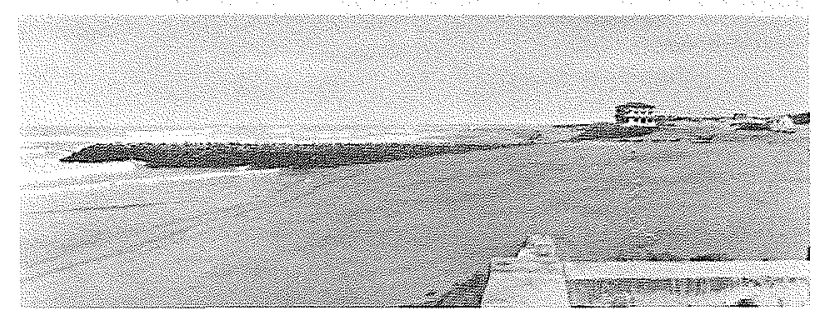

Figure 21 - Plage reformée en nature au Nord des Sables d'Or (1975).

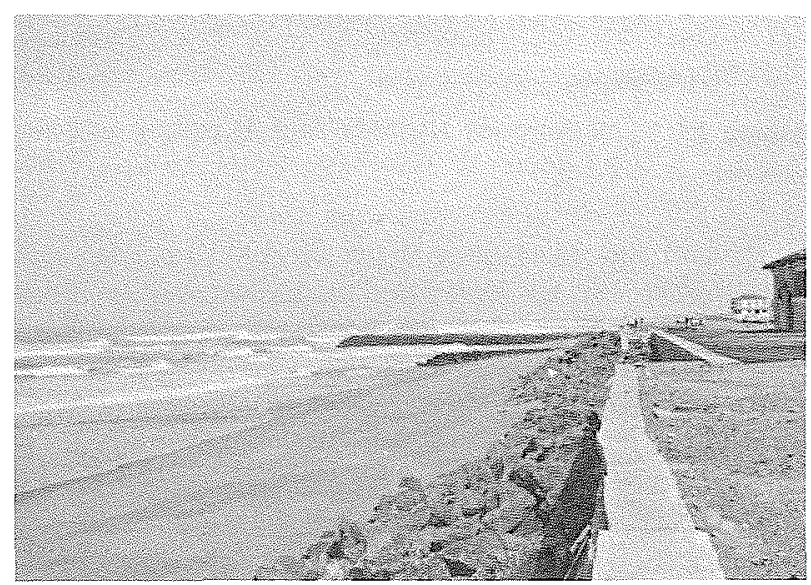

Figure 22 - Plage reformée en nature devant les Sables d'Or (Mars 1976).

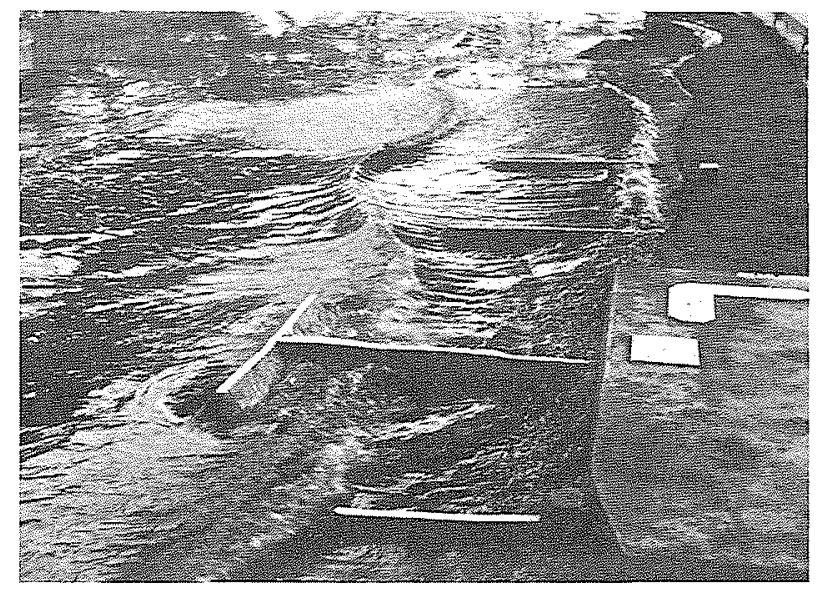

Figure 23 - Epis en $\mathrm{T}$ devant les Sables d'Or, formation locale des plages alvéolaires.

quement supprimée alors que sans ouvrage de défense le recul du littoral aurait atteint $150 \mathrm{~m}$ d'ici l'an 1990 (Figures $20-21-22$ ) ;

- un épi en T devant les Sables d'Or permettrait la formation d'une plage alvéolaire de part et d'autre de l'ouvrage à l'abri du brise-lames; il ne s'agit pas sur le reste du littoral et il faudra associer à cet ouvrage des rechargements en madrague pour compenser les quantités de matériaux se trouvant piégés à l'abri de la barre du T (Figure 23);

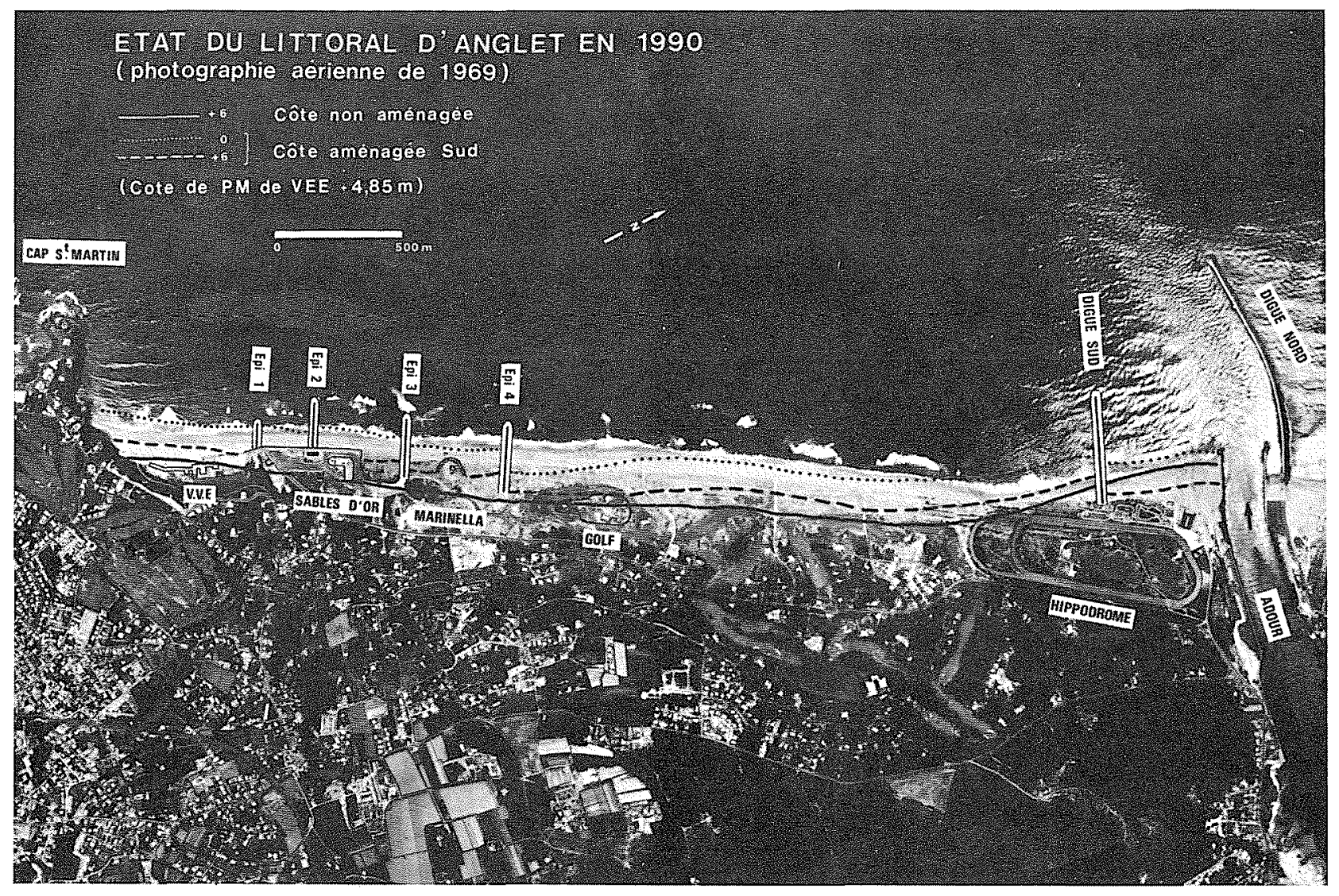

Figure 24 - Etat du littoral d'Anglet pour le modèle en 1990.

(photographic aérienne de 1969) 
- en portant les dépôts de produits de dragages à $200000 \mathrm{~m}^{3}$ par an on ralentit l'érosion des petits fonds par rapport à la période actuelle mais l'isobathe $-4 \mathrm{~m}$ vient quand même tangenter l'extrémité des épis mettant leurs musoirs en danger. Il est formellement conseillé de ne pas réduire ce volume des dépôts et d'essayer, dans toute la mesure du possible, de l'augmenter en profitant des volumes de dragages que l'on sera vraisemblablement amené à extraire dans la partie aval du chenal d'accès au Port de Bayonne pour augmenter les facilités d'accès.

La figure 24 donne l'état prévu par le modèle réduit vers les années 1990 et la figure 25 les aménagements réalisés en nature. Le tableau suivant indique les valeurs du recul du trait de côte en mètres par an dans le cas d'une côte non protégée et protégée.

\begin{tabular}{|l|c|c|c|c|c|c|c|}
\hline \multicolumn{7}{|c|}{ Comparaison du recul du trait de côte en mètres par an } \\
\hline $\begin{array}{l}\text { Distance jetée Sud } \\
\text { en mètres }\end{array}$ & $\begin{array}{c}1200 \\
\text { Hippodrome }\end{array}$ & 1600 & 2000 & $\begin{array}{c}2400 \\
\text { Golf }\end{array}$ & $\begin{array}{c}2800 \\
\text { Marinella }\end{array}$ & 3200 & 3600 \\
\hline Côte non protégée & -2 & -4 & -7 & -10 & -11 & -14 & -14 \\
\hline Côte protégée 1976/82 & $+1,5$ & $-2,8$ & -6 & $-9,5$ & $-4,3$ & $+1,5$ & +1 \\
\hline Côte protégée 1982/88 & $+0,8$ & -1 & -4 & -5 & $-1,5$ & 0 & 0 \\
\hline
\end{tabular}

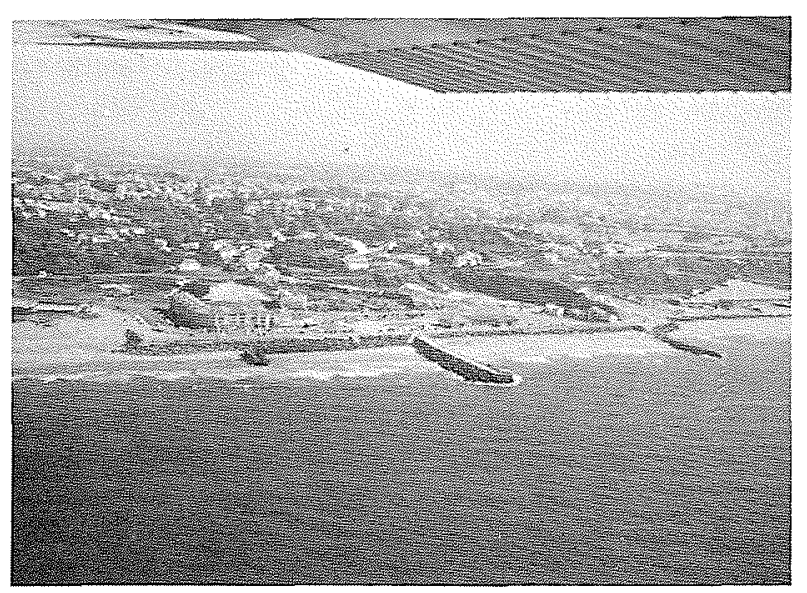

Figure 25 - Vue aérienne en nature des aménagements de la côte d'Anglet.

Dans tous les cas on peut affirmer que sans les aménagements envisagés pour la protection du littoral d'Anglet, la destruction de la côte aurait été inéluctable et que les bâtiments situés au Sud du littoral auraient été très endommagés. En léalisant une digue de $400 \mathrm{~m}$ au Sud de l'embouchure de l'Adour, des épis droits et un épi en $T$ devant les Sables d'Or, en rechargeant le littoral par de la madrague au cours des premières années $\left(210000 \mathrm{~m}^{3}\right.$ dont $70000 \mathrm{~m}^{3}$ de graviers et $140000 \mathrm{~m}^{3}$ de sable très gros) et en alimentant les petits fonds avec plus de $200000 \mathrm{~m}^{3}$ par an de produits de dragages, on arrive à stopper l'érosion du trait de côte dans toute la zone urbanisée et sur plus de $1 \mathrm{~km}$ au Sud de la digue Sud. Entre ces deux zones le littoral continue de s'éroder avec toutefois une décroissance dans le temps et une tendance vers une valeur asymptotique du recul. De même, si l'érosion du trait de côte est réduite, on ne peut sous-estimer celle des petits fonds et les risques qu'elle entraîne pour les musoirs des ouvrages.

Il est probable que si l'on avait pu déverser des quantités de produits de dragages dans la zone des petits fonds, aussi près que possible du bas estran, atteignant le volume total des érosions constatées tant en nature que sur modèle réduit, on aurait pu stopper complètement les phénomènes d'érosion. Une telle suggestion nécessiterait un déversement de $400000 \mathrm{~m}^{3}$ par an environ de sable et des moyens de refoulement à la côte qui semblent très difficiles à mettre en cuvre dans les conditions techniques et économiques actuelles. De même si des impératifs financiers n'avaient pas limité les ouvrages à réaliser il aurait été possible de sauvegarder la zone dite "sauvage" située devant le Golf en s'opposant non seulement aux mouvements latéraux mais aux pertes considérables dans le profil ; cette sug. gestion n'a pu être retenue et n'a pas fait l'objet d'études en modèle réduit. Il est probable que des épis en $T$

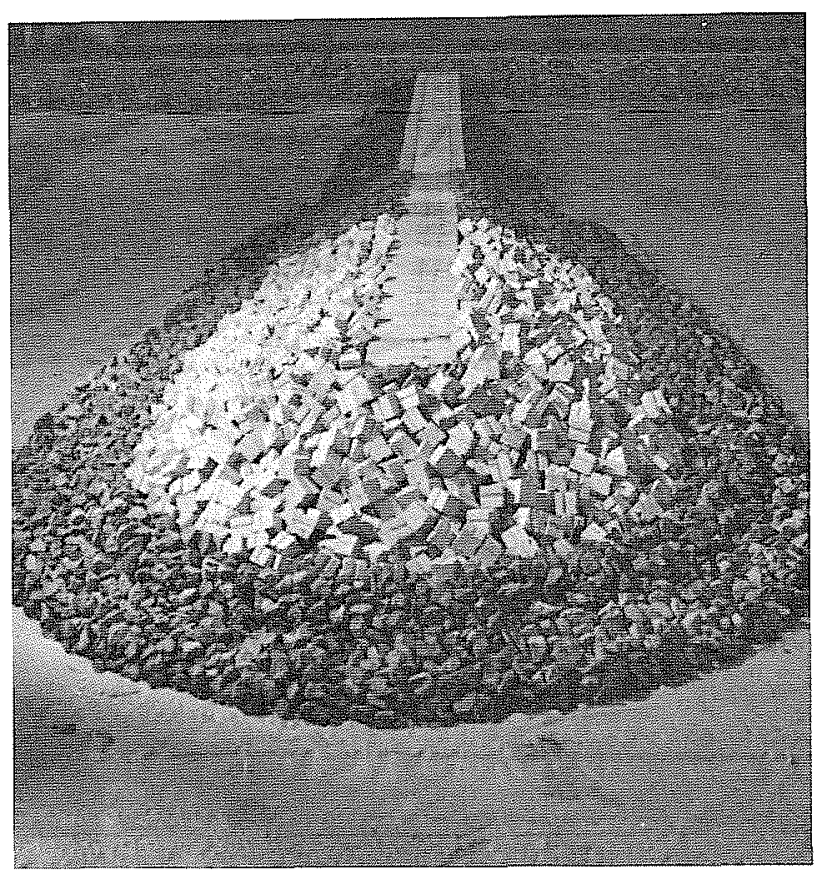

Figure 26 - Structure de la digue réalisée au Sud de l'Adour. 


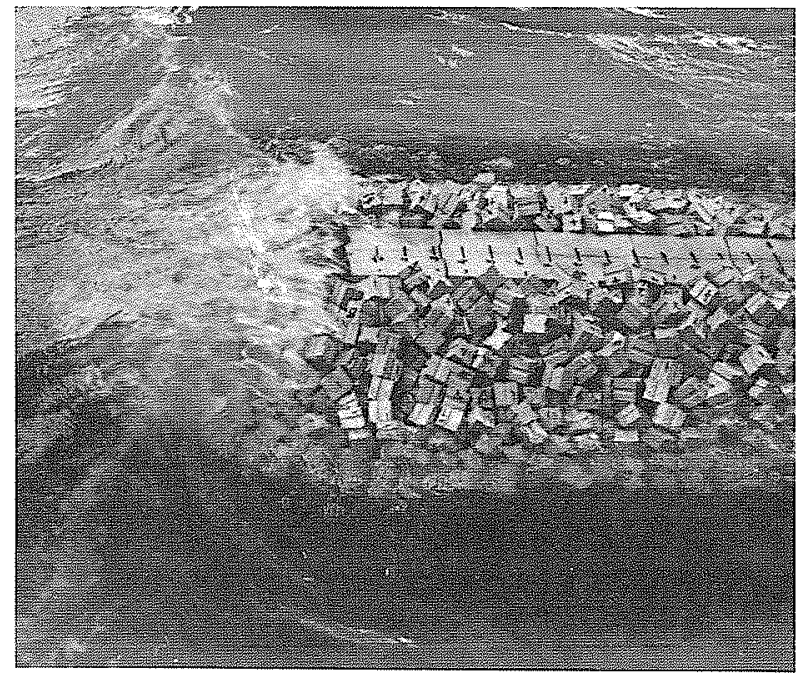

Figure 27 - La digue Sud soumise, en modèle, aux actions des houles.

associés à des rechargements en madrague auraient permis d'améliorer la situation.

Précisons que, parallèlement, des études approfondies ont permis de fixer les caractéristiques optimales de la structure des ouvrages de protection, que ce soit la digue Sud de l'embouchure de l'Adour ou les épis droits des Sables d'Or - Marinella.

On constate que dans cette mer très forte il faut protéger la digue Sud avec des enrochements d'ophite de 8 à 10 tonnes sur un tronçon médian et renforcer le musoir avec des blocs cubiques rainurés de 28 tonnes. Pour ces caractéristiques de blocs la stabilité est bonne mais non surdimensionnée (Figures $26-27$ ).

Pour les épis, réalisés par des profondeurs plus faibles, des enrochements d'ophite de 8 tonnes ne suffisaient pas pour assurer la stabilité de leur tronçon terminal et il est nécessaire de prévoir des blocs de 10 à 12 tonnes.

Tous ces résultats montrent les difficultés qui se présentent devant les travaux à réaliser pour défendre une partie du littoral d'Anglet et la nécessité de ne pas sousestimer la force des houles. Ils montrent également que des espoirs sont permis, sous réserve de réaliser les ouvrages préconisés par les recherches en modèle réduit, pour freiner la dégradation du littoral d'Anglet-Chiberta dans toute la zone urbanisée, rétablir des plages accessibles aux touristes, et améliorer progressivement les accès au Port de Bayonne. 